\title{
Crystallization and degradation behaviour of multiblock copolyester blends in Langmuir monolayers
}

\author{
Victor Izraylit (D), and Yue Liu (D), Institute of Active Polymers, Helmholtz-Zentrum Hereon, 14513 Teltow, Germany; Institute of Chemistry, University of Potsdam, \\ 14476 Potsdam, Germany \\ Natalia A. Tarazona D, Institute of Active Polymers, Helmholtz-Zentrum Hereon, 14513 Teltow, Germany \\ Rainhard Machatschek (D), and Andreas Lendlein (D), Institute of Active Polymers, Helmholtz-Zentrum Hereon, 14513 Teltow, Germany; Institute \\ of Chemistry, University of Potsdam, 14476 Potsdam, Germany \\ Address all correspondence to Andreas Lendlein at Andreas.Lendlein@hereon.de
}

(Received 27 July 2021; accepted 15 September 2021; published online: 15 October 2021)

\begin{abstract}
Supporting the wound healing of soft tissues requires fixation devices becoming more elastic while degrading. To address this unmet need, we designed a blend of degradable multiblock copolymers, which is cross-linked by PLA stereocomplexation combining two soft segments differing substantially in their hydrolytic degradation rate. The degradation path and concomitant structural changes are predicted by Langmuir monolayer technique. The fast hydrolysis of one soft segment leads to a decrease of the total polymer mass at constant physical cross-linking density. The corresponding increase of the average spacing between the network nodes suggests the targeted increase of the blend's flexibility.
\end{abstract}

\section{Introduction}

Synthetic polylactones are polymers, which are produced from cyclic monomers in ring-opening polymerization or from hydroxy acids in polycondensation reactions from natural or synthetic starting materials. Similarities in polymerization mechanisms and polymer structures allow for the preparation of copolymers, where macroscopic properties can be tuned by designing their molecular structure. ${ }^{[1,2]} \operatorname{Poly}(\varepsilon$-caprolactone $)$ (PCL), polylactide (PLA), polyglycolide (PGA), and poly $(p$ dioxanone) are among the few degradable polymers, which are established in clinical applications, such as surgical sutures, scaffolds, or clamps. ${ }^{[3-5]} \mathrm{A}$ change in the mechanical properties of these devices as a consequence of degradation can improve regeneration of the surrounding tissue, if the implant materials become soft and elastic, but do not break.

In our previous work, we have observed that covalently cross-linked copolyesters increase their flexibility, appearing as an increase of elongation at break and decrease of Young's modulus, during degradation, due to an increase in the average chain length between two network nodes. ${ }^{[6]}$ In this study, we hypothesize that a similar effect can be achieved in physical networks. This class of polymeric materials is of particular interest due to the capability of physical intermolecular interactions to be cleaved and reformed with non-destructive processing techniques. This provides broader opportunities for processing of such materials in comparison to covalent networks.

PLA stereocomplexation, which occurs between two opposite stereoisomers poly( $L$-lactide) (PLLA) and poly( $D$-lactide) (PDLA), ${ }^{[7]}$ was selected as a suitable mechanism for the formation of a physical network. PLA stereocomplexes provide higher thermal and mechanical stability than isotactic PLA crystals and require shorter repeating unit sequences to be formed. It has been reported that sequence length of only 7 repeating units was necessary to form PLA stereocomplexes, while homocrystallization was possible in molecules having at least 11 repeating units. ${ }^{[8]}$ This number can even increase for multiblock copolymers with immiscible segments due to the interpenetrating of phases. ${ }^{[9]}$ In this way, it is possible to synthesize a polymeric system, which is incapable of PLA isotactic crystallization, but capable of PLA stereocomplexation after the addition of the opposite stereoisomer. Here, we designed such material as a blend of two multiblock copolymers. The first one consisted of PCL and PLLA segments (PCL-PLLA), where PCL was the main structural component, providing mechanical stability to the designed system. PLLA segments were amorphous and played the role of anchoring points for PLA stereocomplexes. Their length should be limited to 15 repeating units, previously discovered to be capable for stereocomplexation but too short for homocrystallization. ${ }^{[9]}$ This number, however, can differ and should be individually identified for each polymer system. The second component of the blend was a multiblock copolymer with two types of segments. The crystallization of the first type of segments, PDLA, was obstructed by the second component of the multiblock copolymer, which was a random copolymer from diglycolide and $\varepsilon$-caprolactone (PGACL). Here, crystallization was prevented by the random sequence, consequently leaving the PGACL segments highly sensitive to hydrolytic degradation of the PGA repeating units. ${ }^{[10]}$ The molecular design implied that the PGACL segments suppressed stereocomplexation of the neighbouring PDLA segments, when both components were mixed in a blend. 
The multiblock copolymers were synthesized with the previously reported self-polycondensation of reactive diblock precursors procedure, which is capable of achieving high molar mass of the products maintaining accurate control over the sequence structure. ${ }^{[9]}$ The structural changes in the blend during hydrolytic degradation were evaluated with the predictive Langmuir monolayer technique, allowing for in situ and real-time controlling of the material system under accelerated degradation conditions. ${ }^{[11]}$ To determine the crystallization conditions of the individual components in the blend, three methods were combined: (i) the onset of crystallization, detected as a plateau in the $\pi$-A isotherm during compression of the monolayer; (ii) the increase of the complex interfacial viscosity, using interfacial rheology; (iii) the appearance of vibration bands attributed to specific groups in the polymer structure, using Polarization Modulation-Infrared Reflection-Adsorption Spectroscopy (PM-IRRAS). The degradation experiments were performed at constant surface pressure $\pi$, while the changes in the crystalline structure of PCL-PLLA/PGACL-PDLA were investigated with IRRAS and in the rheological experiments.

\section{Materials and methods}

Chloroform (99.9\%, Carl Roth, Karlsruhe, Germany), toluene (99.5\%, Carl Roth, Karlsruhe, Germany), methanol (99\%, Carl Roth, Karlsruhe, Germany), tin(II) 2-hexanoate (Sn(Oct)2) (96\%, Alfa Aesar, Massachusetts, USA), dimethylaminopyridine (DMAP) (99\%, Sigma-Aldrich, Missouri, USA), p-toluenesulfonic acid monohydrate (pTSA) $(98.5 \%$, Sigma-Aldrich, Missouri, USA), anhydrous benzylalkohol (BnOH) (98\%, Acros Organics, Geel, Belgium), 10\% palladium on activated carbon (Alfa Aesar, Massachusetts, USA), deuterated chloroform (99.8\% Sigma-Aldrich, Missouri, USA), diglycolide (99.5\%, Corbion, Amsterdam, Netherlands), L, Ldilactide (99.5\%, Corbion, Amsterdam, Netherlands), D, Ddilactide (99.5\%, Corbion, Amsterdam, Netherlands), and poly( $L$-lactide) PL24 (Corbion, Amsterdam, Netherlands) were used as received. Tetrahydrofuran (THF) (99.9\% Carl Roth, Karlsruhe, Germany), dichloromethane (DCM) (99.9\% Carl Roth, Karlsruhe, Germany), and N,N'-diisopropylcarbodiimide (DIPC) (98\%, Sigma-Aldrich, Missouri, USA) were stored over molecular sieves. $\varepsilon$-Caprolactone (99\%, Acros Organics, Geel, Belgium) was distilled before use. Dimethylaminopyridine p-toluenesolfonate (DMAP.pTSA) was synthesized as reported elsewhere. ${ }^{[12]}$

PDLA-20 was synthesized as reported before. ${ }^{[9]} M_{\mathrm{n}}=2.3 \mathrm{~kg}$ $\mathrm{mol}^{-1} Ð=1.13$ (GPC).

PCL-PLLA was synthesized as reported before. ${ }^{[9]} M_{\mathrm{n}}=200$ $\mathrm{kg} \mathrm{mol}^{-1} Đ=2.1$ (GPC) $l_{\mathrm{CL}}=76$ r.u. $l_{\mathrm{LLA}}=17$ r.u. $\left({ }^{1} \mathrm{H} \mathrm{NMR}\right)$.

PGACL-PDLA. PGACL was synthesized as follows. $4 \mathrm{~g}$ of diglycolide was dried in vacuum at $T_{\text {room }}$ for $1 \mathrm{~h}$ before the reaction inside the reaction vessel. $16.15 \mathrm{~g}$ of distilled CL was added to glycolide with a syringe through a septum under a flow of argon. The mixture was heated up to $140{ }^{\circ} \mathrm{C}$ and stirred for $15 \mathrm{~min}$. Anhydrous THF solutions of $380 \mathrm{mg}$ of $\mathrm{BnOH}$ and
$36 \mathrm{mg}$ of $\mathrm{Sn}(\mathrm{Oct})_{2}$ were added to the reaction mixture with a syringe through a septum under a flow of argon. The reaction lasted for $1 \mathrm{~h}$. Further, the product was cooled to $T_{\text {room }}$ and dissolved in $100 \mathrm{ml}$ of anhydrous chloroform. The product was purified twice by precipitation into n-hexane. Nearly full conversion of diglycolide was achieved. Conversion of CL was $56 \%$ ( ${ }^{1} \mathrm{H}$ NMR). The molar ratio of repeating units $\mathrm{GA} / \mathrm{CL}$ was $48 / 52$, the number average $l_{\mathrm{CL}}=2.1$ r.u. $l_{\mathrm{GA}}=1.1$ r.u. $\left({ }^{13} \mathrm{C} \mathrm{NMR}\right)$ and $M_{\mathrm{n}}=3.9 \mathrm{~kg} \mathrm{~mol}^{-1} Ð=2.1$ (GPC).

PGACL was used as an initiator in PGACL-PDLA diblock copolymer synthesis. This reaction and the following self-polycondensation of PGACL-PDLA diblock macromonomers were performed similarly to PCL-PLLA synthesis. ${ }^{[9]}$ The molar ratio of repeating units GA/CL/LA was 35/38/27, the number average $l_{\mathrm{CL}}=2.4$ r.u. $l_{\mathrm{GA}}=1.7$ r.u. $l_{\mathrm{DLA}}=20$ r.u. $\left({ }^{13} \mathrm{C} \mathrm{NMR}\right)$.

PCL-PLLA/PGACL-PDLA films were prepared with solution casting. Predetermined amounts of PCL-PLLA and PGACL-PDLA were mixed in an equimolar PLA ratio in chloroform with $C=5 \% \mathrm{w} / \mathrm{v}$, poured into a PTFE dish, covered with foil and left to evaporate for $48 \mathrm{~h}$ until no change in mass was registered.

Gel permeation chromatography (GPC) was used to determine number average molecular weights of starting materials and products with a Tosoh EcoSEC HLC-8320 gel permeation chromatograph (Tosoh Bioscience, Stuttgart, Germany) following a procedure described before. ${ }^{[9]}$

Differential scanning calorimetry (DSC) experiments were conducted on a Netzsch DSC 204 Phoenix (Selb, Germany) following a procedure described before. ${ }^{[9]}$

Wide-angle $X$-ray scattering (WAXS) measurements were performed with a D8 Discover spectrometer (Bruker AXS, Karlsruhe, Germany) following a procedure described before. ${ }^{[9]}$

Nuclear magnetic resonance spectroscopy $(N M R){ }^{1} \mathrm{H}$ and ${ }^{13} \mathrm{C}$ NMR spectra were recorded using a DRX Avance 500 $\mathrm{MHz}$ spectrometer (Bruker, Rheinstetten, Germany) at room temperature in deuterated chloroform. Chemical shifts $(\delta)$ are reported in parts per million (ppm) relative to residual chloroform at $\delta 7.26 \mathrm{ppm}$ for ${ }^{1} \mathrm{H}$ NMR spectra and $\delta 77.0 \mathrm{ppm}$ for ${ }^{13} \mathrm{C}$ spectra. Samples were dissolved in $\mathrm{CDCl}_{3}$ at a concentration of $15 \mathrm{mg} \mathrm{mL}^{-1}$.

$\pi$ - $A$ isotherms at the air-water interface were recorded on a PTFE Langmuir trough equipped with Delrin barriers and a level compensation system (KSV LTD, Finland) following a procedure described before. ${ }^{[13]}$ For the calculation of the mean molecular area per a repeating unit (MMA) in multiblock copolymers, an average of the molar mass of the repeating units in the composition was calculated in respect to their molar content.

Polarization-modulated infrared reflection-absorption spectroscopy (PM-IRRAS) was used to determine the crystalline structure of monolayers with a setup from Biolin Scientific (Finland) following a procedure described before. ${ }^{[13]}$ The polymers were spread as $0.15 \mathrm{mg} / \mathrm{ml}$ solutions. The surface pressure $\pi$, at which crystallization occurred, was determined by compressing the monolayer with $1 \mathrm{mN} / \mathrm{m}$ steps with a compression 
rate of $10 \mathrm{~mm} / \mathrm{min}$, followed by recording the IRRAS spectrum after $30 \mathrm{~min}$ of equilibration at the given surface pressure. The ester carbonyl vibration band at wavenumber $\tilde{v}=1724 \mathrm{~cm}^{-1}$ was attributed to PCL, at $\tilde{v}=1755 \mathrm{~cm}^{-1}$ was attributed to isotactic PLA crystals, and at $\tilde{v}=1749 \mathrm{~cm}^{-1}$ was attributed to PLA stereocomplexes. In the degradation experiments, changes in the intensity of a band attributed to PLA stereocomplex crystals were registered while keeping $\pi$ constant and compared against changes in trough area.

Rheology experiments were carried out with an Interfacial Shear Rheometer (IRS, model MCR502) from Anton Paar (Austria) following a procedure described before. ${ }^{[14]}$

Atomic force microscopy $(A F M)$ was used to examine the surface topography and nano-roughness with a MFP-3D (Asylum Research, Santa Barbara, CA, USA) equipped with a silicon cantilever (OLYMPUS OMCL AC160TS-R3). The monolayers were transferred onto a silicon wafer directly from the air-water interface using the Langmuir-Schäfer technique. The scans were evaluated with ImageJ software.

\section{Results and discussion Synthesis of PCL-PLLA/PGACL-PDLA blends}

The design of the PCL-PLLA/PGACL-PDLA blends implies that the isotactic crystallization of PLLA and PDLA segments is suppressed by phase dilution in the nanophase-separated morphology of both multiblock copolymers. PCL-PLLA has been previously reported as a material system, when blended with oligomeric ODLA, performing as a shape-memory polymer actuator, which forms a new class of lactide-based polymers. ${ }^{[9]}$ PGACL-PDLA was synthesized in a manner that PGACL segments possess a highly random sequence structure $\left(l_{\mathrm{CL}}=2.4\right.$ r.u. $l_{\mathrm{GA}}=1.7$ r.u. $)$, therefore, remaining amorphous. The number average sequence length $l_{\text {DLA }}=20$ r.u. of the PDLA segment is sufficient for the formation of isotactic crystals in homopolymers and even in multiblock copolymers. However, PGACL-PDLA formed no crystalline structures observable with WAXS or as melting transitions in DSC. The self-polycondensation reaction was not so effective for PGACL-PDLA diblock copolymers, resulting in a substantially lower number average molar mass when compared to more stable PCL-PLLA diblock copolymers. The reason for this might be the high sensitivity of the PGACL segments in the amorphous domains to hydrolysis.

\section{Crystallization in Langmuir monolayers}

The onset of PDLA crystallization, which would interfere with the formation of stereocomplexes, was determined using a PDLA homopolymer having similar sequence length $l=20$ r.u. with the PDLA segment of PGACL-PDLA. PDLA-20 was synthesized, its compression isotherm was recorded, and its crystallization conditions were studied with interfacial rheology (Figure S1). Isolated PLA crystallites start forming at $\pi>11$ $\mathrm{mN} / \mathrm{m}$, as estimated from the compression isotherm, while pronounced crystallization behaviour occurs at $\pi=20 \mathrm{mN} / \mathrm{m}$ and $\mathrm{MMA}=2.5 \AA^{2}$, as seen from the rheological experiment.

High molar mass PL24 was used as PLA stereocomplex counterpart for PDLA-20 to define the onset of its crystallization. PL24 on its own crystallized at $\pi=5 \mathrm{mN} / \mathrm{m}$, as determined with PM-IRRAS (Figure S2). Here, the vibration band at $\tilde{v}=1751-1754 \mathrm{~cm}^{-1}$ attributed to the carbonyl groups of PLA was identified as a characteristic signal. ${ }^{[15]}$ The fluctuation of the peak position originated from the low signal-to-noise ratio of IRRAS spectra.

Further, the crystallization conditions of an equimolar blend of PL24 and the synthesized PDLA-20 were investigated. The compression isotherm had a two-step profile, suggesting that crystallization occurred in several stages [Fig. 1(a)]. In the equimolar blends, PLA isotactic crystals were formed at $\pi=5$ $\mathrm{mN} / \mathrm{m}$, identified from the band at $\tilde{v}=1754 \mathrm{~cm}^{-1}$, previously observed in PLLA monolayer (Figure S2). Further compression to $\pi=9 \mathrm{mN} / \mathrm{m}$ led to a shift of the carbonyl signal to $\tilde{v}=1749$
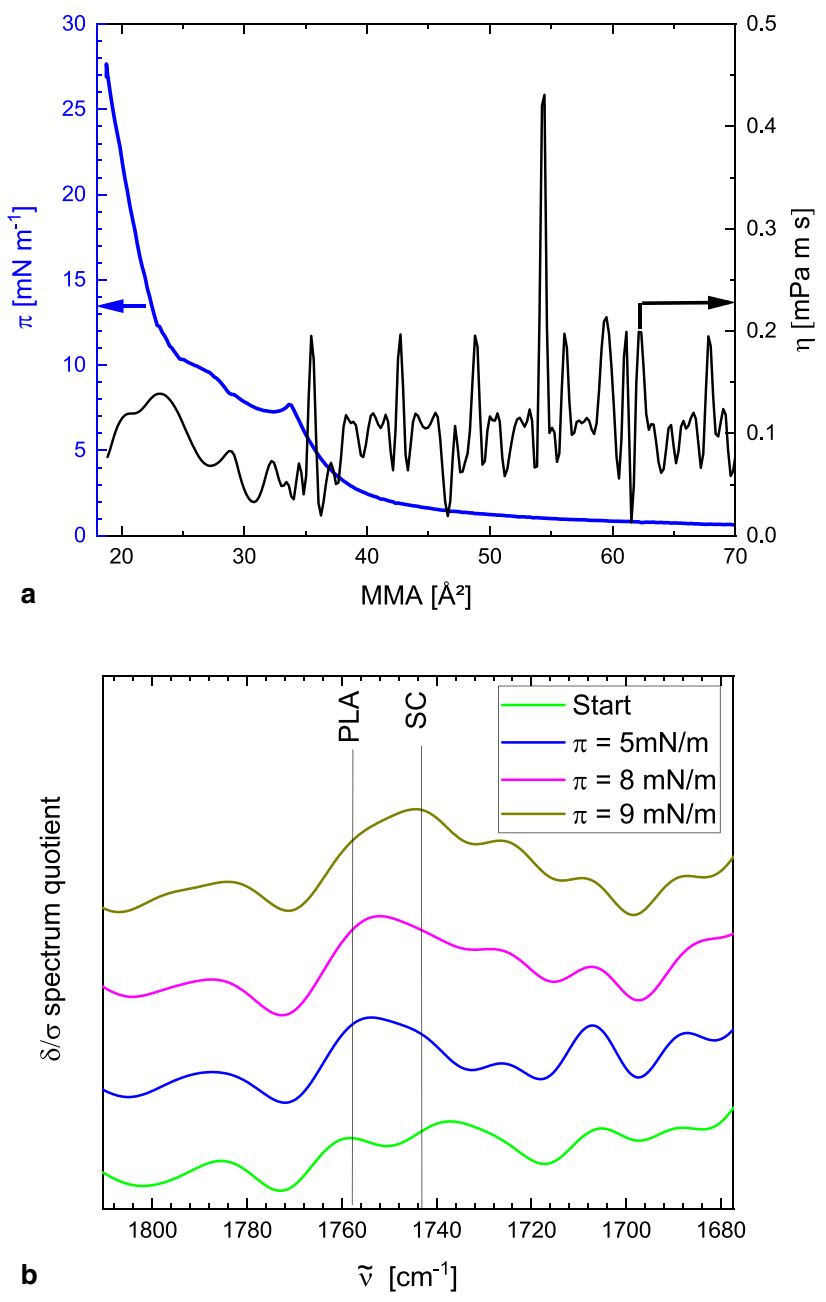

Figure 1. (a) Compression isotherm of PLLA/PDLA and complex interfacial viscosity as a function of MMA in compression of PLLA PDLA monolayer. (b) PM-IRRAS spectra of PLLA/PDLA monolayer at consecutively increased $\pi$. 
$\mathrm{cm}^{-1}$, typically attributed to PLA stereocomplex [Fig. 1(b)]. ${ }^{[15]}$ Interestingly, no increase in the complex interfacial viscosity $(\eta)$ was observed in the rheological experiment at $\pi=5 \mathrm{mN} / \mathrm{m}$ [Fig. 1(a)]. Further, $\eta$ increases scarcely after reaching $\pi=8.4$ $\mathrm{mN} / \mathrm{m}$, and even upon compression to $\pi=25 \mathrm{mN} / \mathrm{m}$, no increase in the interfacial moduli was observed. The reason for this could be hindrances for a collective PLLA crystallization in blends with PDLA. At the same time, small and irregular PLA stereocomplex crystallites, containing low molar mass PDLA, had low mechanical strength and were not able to provide the PLLA / PDLA monolayer with a continuous mechanical stability.

In agreement with the bulk experiments with PCL-PLLA reported before, ${ }^{[9]}$ a PCL-PLLA monolayer was capable of forming only PCL crystallites. This was observed as the appearance of vibration bands in IRRAS spectra at $\tilde{v}=1724 \mathrm{~cm}^{-1}$, attributed to PCL (Figure S3), accompanied by a rapid increase in $\eta$ in the rheological experiment (Figure S3). PM-IRRAS and the rheological experiment gave a similar value of $\pi=10 \mathrm{mN} / \mathrm{m}$ for the onset of PCL crystallization, which is generally in agreement with the previous investigation on PCL homopolymers ${ }^{[16]}$ but is higher than $\pi=9 \mathrm{mN} / \mathrm{m}$ determined for PLA stereocomplex formation. This means that we can adjust the degradation experiment parameters in a way that it is not possible to form any other crystallites in a monolayer except for PLA stereocomplexes. Surprisingly, no increase in interfacial viscosity was observed when compressing the PCL-PLLA/PDLA blend, which was previously reported to have the mechanical properties of a polymeric network in bulk. ${ }^{[17]}$ The reason for this might be the insufficient sensitivity of our rheometer setup. Highly irregular single PLA stereocomplex crystallites formed between oligomeric PDLA and similarly short PLLA segments of PCL-PLLA, disrupted by partial mixing with the PCL phase, most probably did not possess outstanding mechanical properties of high molar mass PLA stereocomplexes. Furthermore, the strict confinement to a two-dimensional monolayer excluded the possibility of intermolecular entanglements, which plays an important role in the mechanical properties of bulk materials. These results correlate with observations made for PLLA/ PDLA blends, where the increase of $\eta$ upon PLA stereocomplexation was barely noticeable [Fig. 1(a)].

\section{Degradation of monolayers}

The PGACL segment is amorphous due to its highly random structure. Hence, its PGA repeating units are highly sensitive to hydrolytic degradation. The average PCL segment length between two PGA segments is smaller than the solubility limit of PCL (ca. hexamer). ${ }^{[18]}$ This means that complete dissolution of the PGACL block in water is possible without cleaving any PCL bonds. The PLA segments of PGACL-PDLA and PCLPLLA, and the PCL segment of the latter are not so sensitive to the uncatalysed hydrolytic degradation; therefore, we can neglect their mass loss in comparison to the PGACL segments. To estimate the degradation rate of PGACL, a hydrolytic degradation experiment of PGACL-PDLA at constant $\pi=5 \mathrm{mN} / \mathrm{m}$ was performed (Figure S4). The point, where the degradation curve reached a plateau, was attributed to the time required for complete degradation of the PGACL segments $t=65 \mathrm{~h}$.

The degradation experiment of PCL-PLLA/PGACL-PDLA $50 / 50 \mathrm{wt} \%$ blend (equimolar by PLA content) was performed at a constant $\pi=9 \mathrm{mN} / \mathrm{m}$ sufficient for PLA stereocomplexation, but too low for PCL crystallization. Isotactic crystallization of PLA in both copolymers was suppressed by shortening its monomer sequences at the synthetic step. Although PCLPLLA/PGACL-PDLA blends showed limited crystallization in bulk, PLA stereocomplexation in monolayers started immediately after reaching $\pi=9 \mathrm{mN} / \mathrm{m}$. The intensity of the $\tilde{v} \approx 1754$ $\mathrm{cm}^{-1}$ band was recorded during the degradation to assess the evolution of the stereocomplex content. The position of the peak was monitored to ensure that no PLA isotactic crystals were formed. The evolution of the intensity of the IR-band attributed to PLA stereocomplexes, together with the reduction of the normalized area $\left(A / A_{0}\right)$ during hydrolytic degradation of PCL-PLLA/PGACL-PDLA blend is presented in Fig. 2. The blend shows the typical sigmoid shape degradation curve of a polymer undergoing a random chain scission, which levels off at $55 \%$ mass loss after ca. $30 \mathrm{~h}$, corresponding to the complete dissolution of PGACL. A further slow linear mass loss
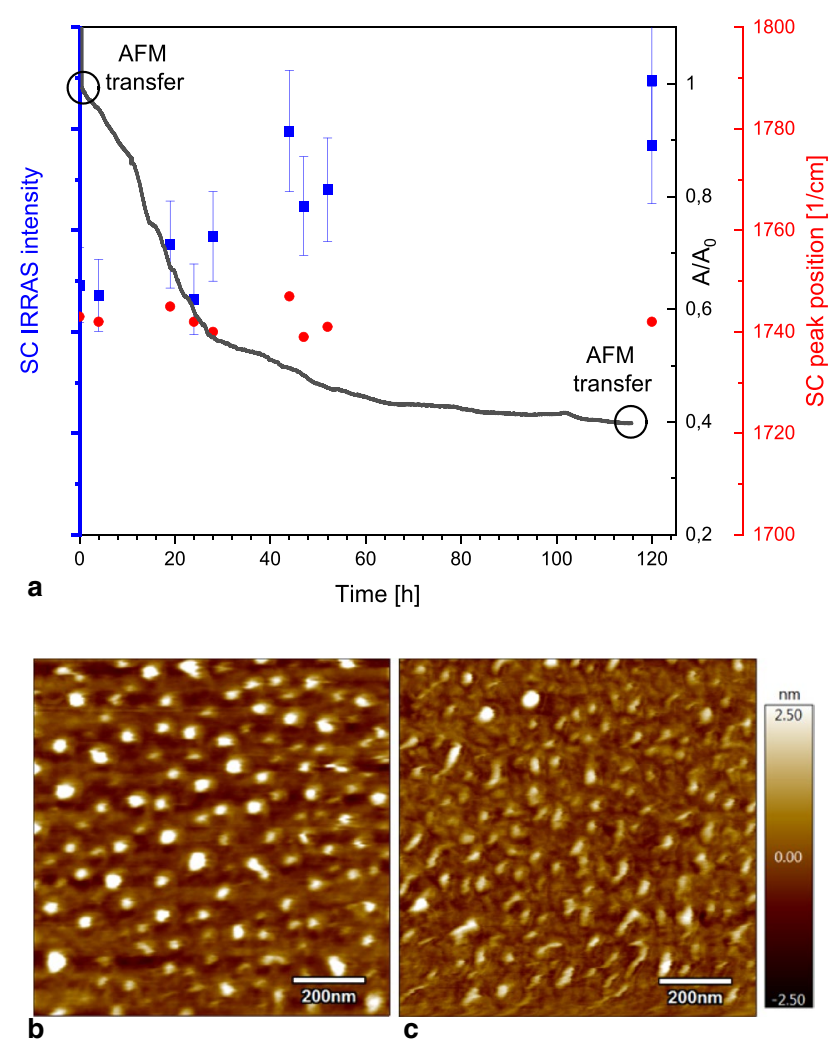

Figure 2. (a) Evolution in time of the intensity and peak position of the IR-band attributed to the carbonyl group vibrations in PMIRRAS experiment during hydrolytic degradation in a monolayer. AFM morphology of the transferred monolayers in the beginning (b) and at the end (c) of the degradation experiment. 
is observed as a consequence of PCL and PLA stereocomplex degradation. An increase in the intensity of the $\tilde{v} \approx 1754 \mathrm{~cm}^{-1}$ band is observed during the degradation. Yet, due to the compression of the layer during degradation, an increase in the areal concentration of stereocomplexes is expected even when no new stereocomplexes are formed. The increase in the PMIRRAS signal can occur due to an increase in PLA stereocomplex crystallinity and due to removal of the degraded material from the monolayer. While we observe approximately a 2.2fold increase in the intensity of the IR-bands, the decrease of film area is 2.5 -fold. This suggests that the degradation of the monolayer happens faster, than new PLA stereocomplexes can form, or the maximal possible crystallinity is reached already at the very beginning of the degradation experiment. These results indicate that the PCL-PLLA/PGACL-PDLA blend maintains its physical cross-linking density throughout the degradation. Amorphous rapidly degrading PGACL segments play no defining role in the mechanical properties of the studied materials, while the pure PCL and PLA domains remain resistant to hydrolysis. Therefore, the increase of the average chain length between two network nodes following the degradation should increase the elasticity of the material at the constant crosslinking density. ${ }^{[6]}$ As the rheometer setup was not capable of registering mechanical properties of the PLA stereocomplex cross-linked monolayer, it was not possible to evaluate changes in elasticity with the selected techniques.

The analysis of monolayer morphology performed with AFM reveals a phase-separated structure, where isolated domains are surrounded with a continuous phase [Fig. 2(b) and (c)]. The isolated domains in both images have similar heights $h=1.24 \pm 0.25 \mathrm{~nm}$, suggesting having a single layer of crystals. ${ }^{[19]}$ As the monolayers were transferred to the silica wafers at $\pi=9 \mathrm{mN} / \mathrm{m}$, only PLA stereocomplex crystallites could be present in these films. Therefore, the isolated domains can be attributed to PLA phases containing stereocomplexes, while the continuous phase consists of amorphous PLA, PCL, and PGACL segments. These results correlate with our previous findings. ${ }^{[20]}$ The degradation of PGACL segments led to changes in the morphology of the PLA domains, suggesting their partial degradation. Further, the surface area fraction of the harder domains attributed to PLA stereocomplex crystallites remained at a constant value of $16 \%$, which supports the finding of the Langmuir degradation experiment that the cross-linking density does not decrease during the hydrolysis.

\section{Conclusion}

In this work, a multiblock copolymer with poly( $D$-lactide) and poly[glycolide-ran-( $\varepsilon$-caprolactone)] segments was created, where crystallization of the GA and CL sequences is suppressed by the highly random sequence structure, and isotactic crystallization of the PDLA segments is not possible due to insufficient sequence length. Limited miscibility of PGACL-PDLA and PCL-PLLA in chloroform hindered PLA stereocomplexation in bulk, while the transfer to a two-dimensional monolayer facilitated formation of PLA stereocomplexes through improving miscibility of the multiblock copolymers. The molecular design of the PGACL-PDLA/PCL-PLLA blends enabled controlling the hydrolytic degradation behaviour, leading to molar mass decrease, while keeping cross-linking density, i.e. PLA stereocomplex $\varphi_{\mathrm{c}}$ constant. The attributed increase of the average chain length between two network nodes should lead to the improvement of the elastic properties in bulk. The developed material provides a perspective platform for the development of medical implants with adjustable properties during the degradation.

\section{Acknowledgments}

The authors thank Ms. Manuela Keller for her support in the monolayer degradation laboratory. This work was financially supported by the Helmholtz Association through programmeoriented funding.

\section{Funding}

Open Access funding enabled and organized by Projekt DEAL.

\section{Declarations}

\section{Conflict of interest}

The authors declare no conflict of interest.

\section{Data availability}

Data will be made available on reasonable request.

\section{Open Access}

This article is licensed under a Creative Commons Attribution 4.0 International License, which permits use, sharing, adaptation, distribution and reproduction in any medium or format, as long as you give appropriate credit to the original author(s) and the source, provide a link to the Creative Commons licence, and indicate if changes were made. The images or other third party material in this article are included in the article's Creative Commons licence, unless indicated otherwise in a credit line to the material. If material is not included in the article's Creative Commons licence and your intended use is not permitted by statutory regulation or exceeds the permitted use, you will need to obtain permission directly from the copyright holder. To view a copy of this licence, visit http://creativecommons. org/licenses/by/4.0/.

\section{Supplementary Information}

The online version contains supplementary material available at https://doi.org/10.1557/s43579-021-00107-y. 


\section{References}

1. K. Madhavan Nampoothiri, N.R. Nair, R.P. John, An overview of the recent developments in polylactide (PLA) research. Bioresour. Technol. 101, $8493(2010)$

2. M. Balk, M. Behl, C. Wischke, J. Zotzmann, A. Lendlein, Recent advances in degradable lactide-based shape-memory polymers. Adv. Drug Deliv. Rev. 107, 136 (2016)

3. A. Lendlein, R. Langer, Biodegradable, elastic shape-memory polymers for potential biomedical applications. Science 296, 1673 (2002)

4. R.P. Brannigan, A.P. Dove, Synthesis, properties and biomedical applications of hydrolytically degradable materials based on aliphatic polyesters and polycarbonates. Biomater. Sci. 5, 9 (2016)

5. F. Asghari, M. Samiei, K. Adibkia, A. Akbarzadeh, S. Davaran, Biodegradable and biocompatible polymers for tissue engineering application: a review. Artif. Cells Nanomed. Biotechnol. 45, 185 (2017)

6. A.T. Neffe, G. Tronci, A. Alteheld, A. Lendlein, Controlled change of mechanical properties during hydrolytic degradation of polyester urethane networks. Macromol. Chem. Phys. 211, 182 (2010)

7. H. Tsuji, Poly(lactic acid) stereocomplexes: a decade of progress. Adv. Drug Deliv. Rev. 107, 97 (2016)

8. S.J. de Jong, W.N.E. van Dijk-Wolthuis, J.J. Kettenes-van den Bosch, P.J.W. Schuyl, W.E. Hennink, Monodisperse enantiomeric lactic acid oligomers: preparation, characterization, and stereocomplex formation. Macromolecules 31, 6397 (1998)

9. V. Izraylit, O.E.C. Gould, T. Rudolph, K. Kratz, A. Lendlein, Controlling actuation performance in physically cross-linked polylactone blends using polylactide stereocomplexation. Biomacromol 21, 338 (2020)

10. H.K. Makadia, S.J. Siegel, Poly Lactic-co-glycolic acid (PLGA) as biodegradable controlled drug delivery carrier. Polymers (Basel) 3, 1377 (2011)

11. R. Machatschek, B. Schulz, A. Lendlein, Langmuir monolayers as tools to study biodegradable polymer implant materials. Macromol. Rapid Commun. 40, 1800611 (2019)
12. J.S. Moore, S.I. Stupp, Room temperature polyesterification. Macromolecules 23, 65 (1990)

13. N.A. Tarazona, R. Machatschek, B. Schulz, M.A. Prieto, A. Lendlein, Molecular insights into the physical adsorption of amphiphilic protein PhaF onto copolyester surfaces. Biomacromol 20, 3242 (2019)

14. N.A. Tarazona, R. Machatschek, A. Lendlein, Influence of depolymerases and lipases on the degradation of polyhydroxyalkanoates determined in langmuir degradation studies. Adv. Mater. Interfaces 7, 2000872 (2020)

15. J. Zhang, H. Sato, H. Tsuji, I. Noda, Y. Ozaki, Differences in the $\mathrm{CH} 3 \cdots 0=\mathrm{C}$ interactions among poly(L-lactide), poly(L-lactide)/poly(D-lactide) stereocomplex, and poly(3-hydroxybutyrate) studied by infrared spectroscopy. J. Mol. Struct. 735-736, 249 (2005)

16. N. Hasan, C. Fuchs, C. Schwieger, K. Busse, 0. Dolynchuk, J. Kressler, Crystallization of poly( $\varepsilon$-caprolactone) at the air-water interface studied by IRRAS and GI-WAXS. Polymer 196, 122468 (2020)

17. V. Izraylit, M. Heuchel, O.E.C. Gould, K. Kratz, A. Lendlein, Strain recovery and stress relaxation behaviour of multiblock copolymer blends physically cross-linked with PLA stereocomplexation. Polymer 209, 122984 (2020)

18. M. Hakkarainen, G. Adamus, A. Höglund, M. Kowalczuk, A.-C. Albertsson, ESI-MS reveals the influence of hydrophilicity and architecture on the water-soluble degradation product patterns of biodegradable homo- and copolyesters of 1,5-dioxepan-2-one and $\varepsilon$-caprolactone. Macromolecules 41, 3547 (2008)

19. K. Watanabe, J. Kumaki, Extended-chain crystallization and stereocomplex formation of polylactides in a Langmuir monolayer. Polym. J. 52, 601 (2020)

20. V. Izraylit, O.E.C. Gould, K. Kratz, A. Lendlein, Investigating the phasemorphology of PLLA-PCL multiblock copolymer/PDLA blends cross-linked using stereocomplexation. MRS Adv. 5, 699 (2019) 